\title{
Process design and simulation of a flexible MEMS array antenna
}

\author{
Lin Cong ${ }^{1, a}$, Lixin $\mathrm{Xu}^{1, b}$, Qi Han ${ }^{1, c}$ and Tao Dong ${ }^{2, d}$ \\ ${ }^{1}$ School of Mechatronical Engineering, Beijing Institute of Technology, Beijing 100081, China; \\ ${ }^{2}$ Space Star Technology Company Limited, Beijing 100086, China. \\ acconglin_MEMS@163.com, ' Ixxu@bit.edu.cn, chanqi1988@bit.edu.cn, ${ }^{\mathrm{d}}$ dongtaoandy@163.com
}

Keywords: MEMS, LCP, process, simulation.

\begin{abstract}
It is important to develop the micro-electro-mechanical system (MEMS) fabrication technology based on polymers. Liquid crystal polymer (LCP) is a suitable substrate for fabricating the flexible MEMS array antenna applied to conforming and bending conditions. This paper presents the metallization process design of the LCP substrate and the patterned process design of the flexible MEMS array antenna. Moreover, by process simulation with the software COVENTOR the process design of the flexible MEMS array antenna has been verified to be feasible.
\end{abstract}

\section{Introduction}

Micro-electro-mechanical system (MEMS) is a kind of miniature system, which utilizes integrated circuit (IC) manufacturing together with micro-architecture technologies to implement the integration of micro-structures , micro-sensors, micro-actuators, control/processing circuits and even interfaces, communication, power supplies etc.[1] Materials used in the MEMS are very extensive which include silicon and its compounds, non-silicon semiconductors, metal, memory alloy, glass, ceramic, and polymer.[2] Furthermore it is worth noting that polymer is widely used for smart skin and wearable devices[3,4] since its characteristics of flexibility, biological compatibility and corrosion resistance. Therefore it is important to develop the MEMS fabrication technology based on polymers.

Liquid crystal polymer (LCP) is a high molecule polymer material and it is a suitable substrate for fabricating the flexible MEMS array antenna applied to conforming and bending conditions[5]. DeJean[6] designed microstrip antenna arrays that can be integrated with remote sensing applications, and proposed a thin seed layer of titanium to improve the stiction between copper and LCP. Pavuluri[7] presented a MEMS-based fabrication process to obtain precise metal patterns on substrates with the aim of reducing metal losses at high frequencies. Wang[8] proposed radio frequency (RF) MEMS antenna arrays with multilayer structures and used a standard photolithographic process for all the fabrication.

This paper presents the process design and simulation of a flexible MEMS array antenna. The metallization process of the LCP substrate and the patterned process of the flexible MEMS array antenna have been designed. Moreover, by process simulation with the software COVENTOR the process design of the flexible MEMS array antenna has been verified to be feasible.

\section{Process Design}

Metallization Process of the LCP Substrate. Using the positive photoresist (Fig. 1(a)), the LCP substrate sticks to the silicon wafer before the metallization process, which makes the substrate flat for next processes. Since the surface of LCP features low energy, chemical inertness, and weak boundary layer, which can lead to difficulties in depositing metal directly on it such as the poor adhesion between LCP and copper. The low-temperature plasma etching technology (Fig. 1(b)) is used as the surface treatment method of LCP in the metallization process, and such a roughing treatment improves the stickability of LCP and copper. The steps of the low-temperature plasma etching technology are as follow. First, use acetone and ultrapure water to clean the LCP substrate 
and then dry it out. Next, the LCP substrate fixed on the plate is pushed into the plasma container and placed between the two electrodes. At last, when the vacuum pump and the RF power are turned on, the LCP substrate is etched by oxygen plasmas.

After the surface treatment, the metal-clad LCP board which includes chromium layer and copper layer (Fig. 1(c)) is fabricated by the electroplating method. The chromium layer is formed on the surface of LCP by sputtering coating as an adhesive layer to improve the peeling strength. Besides, the copper layer is deposited on the chromium layer using sputtering coating as a seed layer. Then the copper layer is increased to a desired thickness through electroplating. In particular, the spluttering coating uses cations generated by glow discharge to bombard the cathode target surface at high speed in the electric field, and the spurting out atoms or molecules are deposited on the substrate forming films. Such films using spluttering coating have excellent substrate adhesiveness with high density and small pinholes. Moreover, films with better uniformity and higher precision can be obtained by controlling spluttering time and temperature precisely. Next, the LCP substrate is peeled off from the silicon wafer in acetone solution (Fig. 1(d)) when one side metallization of the LCP substrate is complete. Then the metallization of the other side of LCP is fabricated in the same way (Fig. 1(e)). The metallization process design of the LCP substrate is shown in Fig. 1.

Patterned Process of the Antenna. The patterned process of the antenna is developed by lithography and chemical etching on the basis of the metallization process of the LCP substrate. Lithography is a graphic reproduction technology using the selective light radiation of the photoresist layer to change its chemical properties and removing the corresponding parts, which completes the pattern transferring from the mask to the substrate surface. The graphic obtained by lithography can acts as the mask of the follow-up process. Moreover, the steps of lithography (Fig. 2 (a) (b)) include spin coat, pre-bake, exposure, development and post-bake, and detailed discussion about the steps are given respectively as follow: (1) The silicon wafer is absorbed to the centrifugal spin plate by vacuum with spinning at high speed to form a uniform photoresist layer on the surface of copper. (2) The solvent of the photoresist is evaporated to cure the photoresist on the copper layer. (3) The photoresist is exposed to light to obtain the graphic after alignment with the mask and the silicon. (4) The silicon is put in the developer to dissolve and remove the parts of photoresist modified by light. (5) The solvent of the photoresist is evaporated further to improve the mask effect of the photoresist layer.After the lithography process the next process is the chemical etching (Fig. 2 (c) (d)). Since the metallization layer of the LCP substrate includes chromium layer and copper layer. The chromium layer is etched by ammonium ceric nitrate, and the copper layer is etched by ammonium persulfate. Furthermore, using acetone solution the photoresist is removed, and the LCP substrate is peeled off from the silicon wafer (Fig. 2 (e)). The patterned process design of the antenna is shown in Fig. 2.

(a) LCP sticking to the silicon wafer

(b) the low-temperature plasma etching of LCP

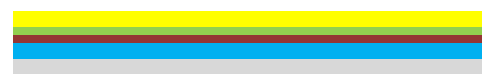

(c) sputtering $\mathrm{Cr}$, sputtering $\mathrm{Cu}$ and electroplating $\mathrm{Cu}$

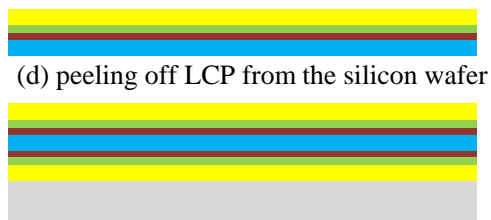

(e) repetition of $(\mathrm{a}) \sim(\mathrm{c})$ on the other side of LCP

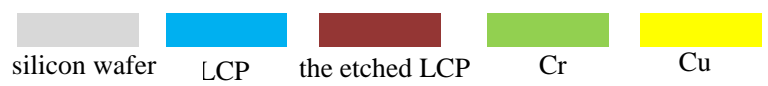

Fig. 1. The metallization process design of LCP

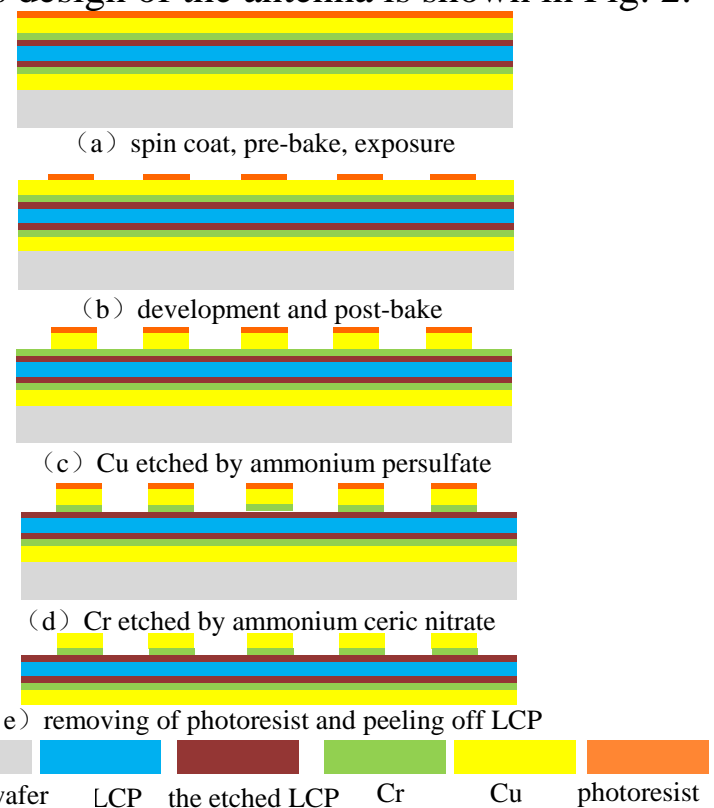

silicon wafer LCP the etched LCP $\mathrm{Cr} \quad \mathrm{Cu}$ photoresist

Fig. 2. The patterned process design of antenna 


\section{Process Simulation}

The fabrication process of the flexible MEMS array antenna is simulated by using the software COVENTOR, and its flow chart is shown in Fig. 3.

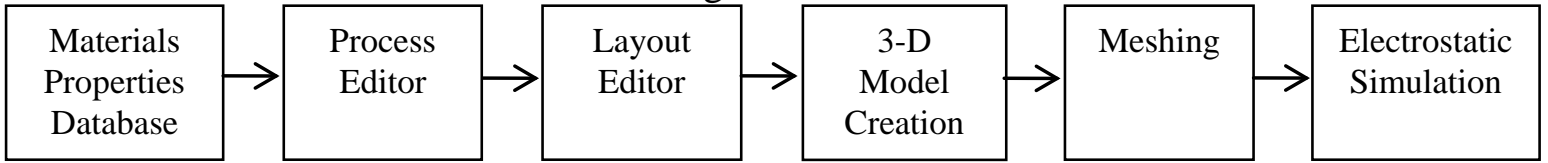

Fig. 3. The flow chart of the process simulation.

The properties of materials used in the flexible MEMS array antenna are added to the database as shown in Table 1.The steps of the fabrication process are filled in the process editor, and the table of the process design is shown in Table 2.

Table 1 Properties of Materials

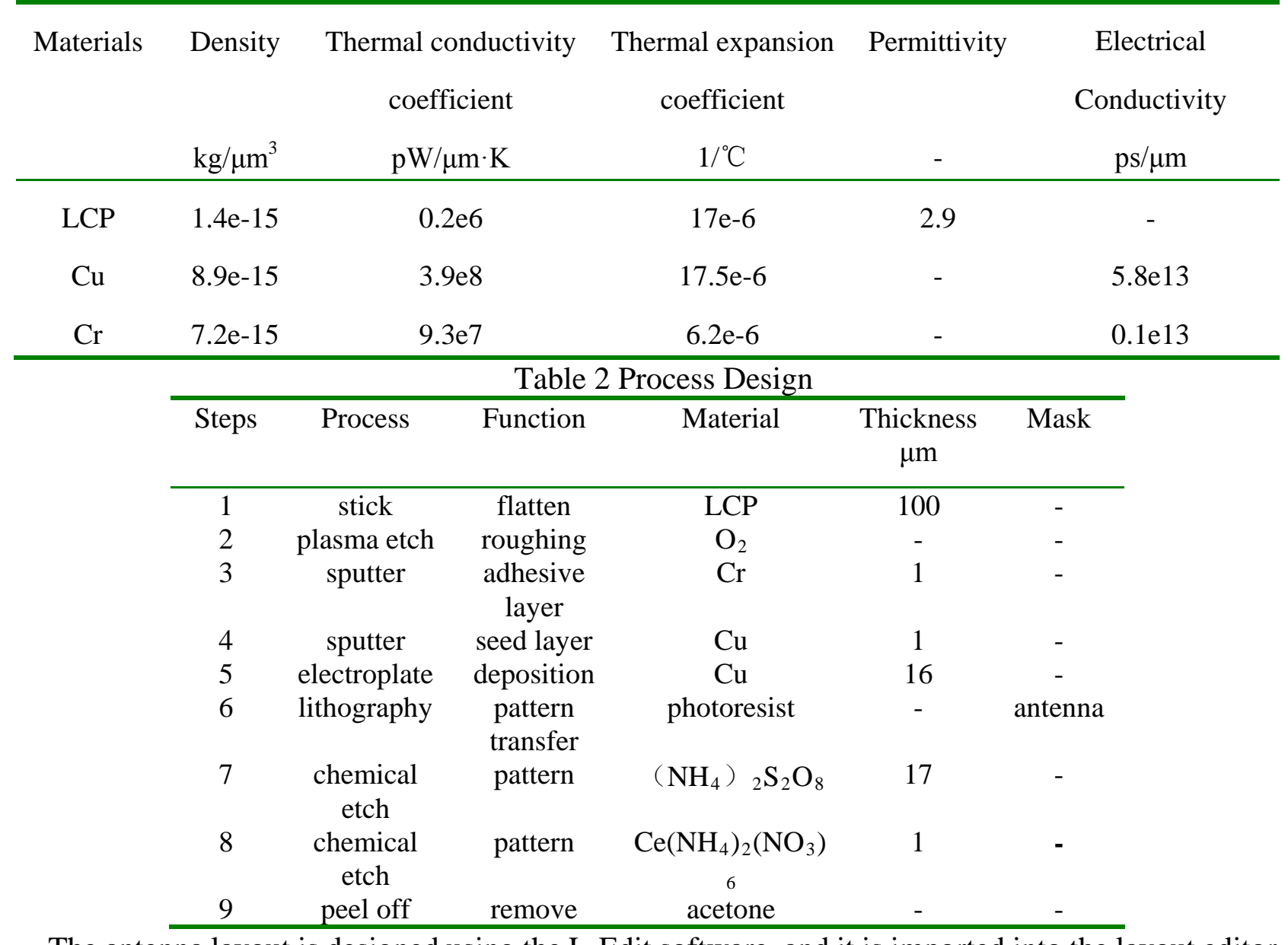

The antenna layout is designed using the L-Edit software, and it is imported into the layout editor. L-Edit is a module with the IC layout edition function and the automatic routing function. When creating the graphics file with L-Edit, the consumer unit is set as millimeter and it is equal to 1000 internal units to ensure the precision of the graphics. The layout of the flexible MEMS array antenna is drawn as shown in Fig. 4.

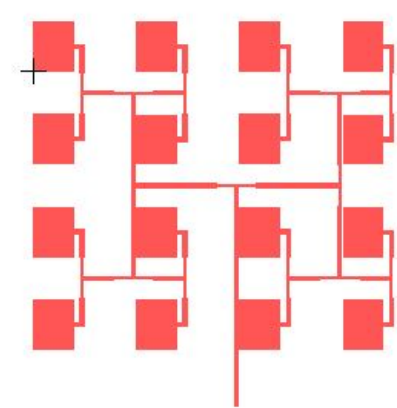

Fig. 4. The layout of the antenna

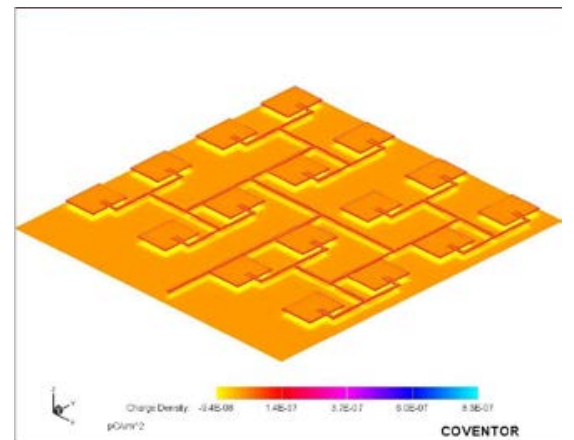

Fig. 5. The simulation result of the charge density 
According to the process design in the process editor and the antenna layout in the layout editor, 3-dimention model of the flexible MEMS array antenna is generated in the preprocessor. The 3-dimention model is meshed by using hexahedron units. Further, the simulation of the grid antenna is carried out in the electrostatic field module. The input voltage of the antenna ground layer is $0 \mathrm{~V}$, and the input voltage of the antenna patch layer is $1 \mathrm{~V}$. The simulation result shows the charge of the ground layer is $-23 \mathrm{pC}$ and the charge of the patch layer is $23 \mathrm{pC}$. The simulation result of the charge density in the antenna with hiding the LCP substrate is shown in Fig. 5. The charge density of the ground layer and the patch layer are the same, which indicates a good connection of the conductor layer.

\section{Summary}

This paper presented the metallization process design of the LCP substrate and the patterned process design of the flexible MEMS array antenna. By using the low-temperature plasma etching technology in the metallization process the poor adhesion between LCP and copper has been improved. The chromium layer was proposed to be an adhesive layer to improve the peeling strength between LCP and copper. Moreover, the process simulation was carried out using the software COVENTOR to demonstrate that the fabrication process of the antenna is available, which supplies guarantees for the fabrication of the flexible MEMS array antenna.

\section{Acknowledgment}

This work is funded by the Chinese Academy of Space Technology under grant CAST 2014-06, and authors gratefully acknowledge the support from it. The corresponding author email address is conglin_MEMS@163.com.

\section{References}

[1] Y.Chen, Development and prospect of micro-electromechanical system, Electro-Mechanical Engineering. 27 (2011) 1-7.

[2] Z. Y. Wang, Microsystem design and fabrication, first ed., Beijing, 2008.

[3] Y. Li, W. P. Li, and X. G. Zhu, Design and fabrication of flexible MEMS anti-drag skin, Optics and Precision Engineering. 20 (2012) 2696-2703.

[4] D. D. Cara, J. Trajkovikj, R. Torres-Sanchez, A low profile UWB antenna for wearable applications the tripod kettle antenna, IEEE 7th European Conf. on Antennas and Propag., Gothenburg. (2013) 3257-3260.

[5] M. Ur-Rehman, Q. H. Abbasi, and M. Akram, Design of band-notched ultra wideband antenna for indoor and wearable wireless communications, IET Microwave Antennas Propag.. 9(2015) 243-251.

[6] G. De Jean, R. Bairavasubramanian, D. Thompson, Liquid crystal polymer (LCP): a new organic material for the development of multilayer dual-frequency /dual-polarization flexible antenna arrays, IEEE Antennas and Wireless Propag. Lett.. 4(2005) 22-26.

[7] G. Wang, R. Bairavasubramanian, B. Pan, Radiofrequency MEMS-enabled polarization-reconfigurable antenna arrays on multilayer liquid crystal polymer, IET Microwaves Antennas and Propag.. 13(2011) 1594-1599.

[8] S. K. Pavuluri, C. Wang, A. J. Sangster, High efficiency wideband aperture-coupled stacked patch antennas assembled using millimeter thick micromachined polymer structures, IEEE Transactions on Antennas and Propag.. 58(2010) 3616-3621. 